\title{
Review of hygiene adaptations among UK doctors in controlling the spread of SARS-CoV-2 infection
}

\author{
Authors: Syed Ammar Husain, ${ }^{\mathrm{A}}$ Syed Arshad Husain, ${ }^{\mathrm{B}}$ Obaid $\cup$ Khan, ${ }^{\mathrm{C}}$ Leon D'Cruz ${ }^{\mathrm{D}}$ and Victoria Allgar ${ }^{\mathrm{E}}$
}

\section{Background}

A significant number of healthcare workers around the world have contracted COVID-19 from their workplace, thus there is a need to investigate common hygiene practices.

Aim

We aimed to describe the common hygiene adaptations of doctors in the hospital and at home.

\section{Methods}

A survey of 110 doctors in UK was carried out to determine their hygiene adaptations and practices. Data were collected on demographics and personal protective equipment compliance.

Results

The key findings were frequent hand washing (51\%), change of clothing entering and leaving hospital ( $88 \%$ and $92 \%$, respectively), taking a shower upon returning home (85\%) and washing work attire at temperatures of $60-80^{\circ} \mathrm{C}(50 \%)$. A higher proportion of junior doctors washed their scrubs $(p=0.004)$ and stethoscopes $(p=0.014)$ compared with consultants and seniors. Female doctors cleaned their mobile phones $(p=0.022)$ and work belongings $(p=0.01)$ more frequently.

\section{Conclusion}

This study signified that junior doctors were more meticulous in hygiene adaptations and female doctors were more fastidious in personal hygiene. The observations of this study may be beneficial in preventing transmission of infection to families of healthcare professionals and are important to implement in the case of a second wave of COVID-19.

KEYWORDS: COVID-19, hygiene practices, hygiene adaptations, prevention, UK frontline doctors

DOI: $10.7861 /$ clinmed.2020-0724

Authors: Ayear-3 medical student, Hull York Medical School, York, UK; ${ }^{B}$ respiratory consultant physician, Maidstone and Tunbridge Wells NHS Trust, Maidstone, UK and honorary senior clinical lecturer,

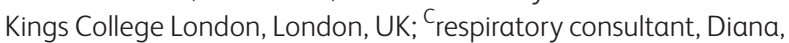
Princess of Wales Hospital, Grimsby, UK; D research associate, Queen Alexandra Hospital, Portsmouth, UK; ${ }^{E}$ head of Centre for Health and Population Sciences, Hull York Medical School, York, UK

\section{Background}

The coronavirus (SARS-CoV-2) is an insidious spreading ribonucleic acid (RNA) virus, that has devastated the world with high mortality figures. ${ }^{1}$ Healthcare workers (HCW) have an increased risk of contracting the virus $9.6 \%$ (odds ratio (OR) 2.03) in their working environment. ${ }^{2}$ As a result, a significant proportion of deaths worldwide have been healthcare workers.

COVID-19 is a serious disease, it was first found to cause pneumonia with very high rates of deaths in Wuhan, China, in 2019. 'The World Health Organization (WHO) declared COVID-19 as a pandemic. ${ }^{3}$

SARS-CoV-2 is a difficult virus to control and was found to 'remain viable and infectious in aerosols for hours and on surfaces for days. ${ }^{4}$ COVID-19 can spread rapidly and with ease, which highlights a cause of concern for those dealing with patients with COVID-19. In light of the rapid progression in new COVID-19 cases, it is crucial for healthcare workers to take more responsibility in reducing the potential spread of COVID-19.

This study was aimed at investigating the hygiene adaptations of doctors to reduce the spread of the SARS-CoV-2 infection onto their families and others in the UK.

\section{Aims}

We had three core objectives.

$>$ To determine the compliance and awareness of personal protective equipment (PPE) and the relevant equipment being used in different environments.

> To describe the common hygiene practices of UK doctors in the hospital settings and their home at the time of the COVID-19 pandemic.

> To compare the hygiene adaptations based on the following environments.

> Respiratory high dependency unit or intensive care unit involved in the direct care of patients with COVID-19 in possibly aerosolised environments (AE_COVID).

> Respiratory units or general medical ward involved in the direct care of patients with COVID-19 in a non-aerosolised environment (NAE_COVID).

> General medical ward that is not usually directly working with patients with COVID-19 (NON_COVID).

\section{Methods}

The study received ethical approval from the Hull York Medical School Ethics Committee (reference: 20 26; 28 May 2020). The 
Table 1. Inclusion and exclusion criteria of the study

$\begin{array}{lll}\text { Study characteristic } & \text { Inclusion criteria } & \text { Exclusion criteria } \\ \text { Study design } & \begin{array}{l}\text { Descriptive study } \\ \text { Cross-sectional survey }\end{array} & \\ \text { Population } & \begin{array}{l}\text { Secondary and tertiary care doctors of all medical grades } \\ \text { working in the time of the COVID-19 pandemic }\end{array} & \begin{array}{l}\text { Other healthcare workers and those who are } \\ \text { not working in secondary and tertiary care }\end{array} \\ \text { Location } & \text { UK } & \text { Outside the UK } \\ \text { Clinical settings } & \begin{array}{l}\text { Respiratory high dependency unit or intensive care unit } \\ \text { involved in the direct care of patients with COVID-19 in an } \\ \end{array} & \text { Norosolised environment (AE_COVID) } \\ & \begin{array}{l}\text { Respiratory unit or general medical ward involved in } \\ \text { the direct care of patients with COVID-19 in the non- }\end{array} & \\ & \begin{array}{l}\text { aerosolised environment (NAE_COVID) } \\ \text { General medical ward that are not usually directly in care } \\ \text { of patients with COVID-19 (NON_COVID) }\end{array} & \end{array}$

study was conducted as a questionnaire survey using Qualtrics and distributed electronically among doctors of all grades in secondary and tertiary care dealing with patients with COVID-19, using email, social media (including Hull York Medical School Alumni Association) and professional colleagues known to the authors. ${ }^{5}$

The inclusion/exclusion criteria for the recruitment of participants to the study are shown in Table 1. Informed consent was obtained from all participants at the beginning of the survey. The study commenced at the first wave of the COVID-19 pandemic. A pilot study was carried out initially on test participants to review responses.

As shown in supplementary material S1, the survey included questions on demographics, compliance and awareness of PPE, hygiene practices at the workplace (hospital), and hygiene practices at home.

Doctors working in the AE_COVID were based on the respiratory dependency care units or intensive care. These environments are noted for higher levels of aerosolised particles in the environment. Doctors working in the NAE_COVID environment were based in the respiratory department or general medical wards where the contribution of aerosolised particles was minimal in the environment.

Junior doctors were classed as foundation year-1-2 trainees (FY1-2), internal medical trainees (IMT1-2), specialist trainees (ST1-2) and the rest were classified as senior doctors (specialist trainees 3-8 (ST3-8), associate specialists, clinical assistants (middle-grade doctors) and consultant grades).

Responses to our questions were mixed, using a Likert scale between $0-5$ ( 0 being the lowest measured outcome and 5 with the highest measured outcome) while others were either single 'yes/no' responses or a free-form text response which allowed a longer subjective response from participants.

\section{Statistical analysis}

Descriptive statistics were presented as the mean (standard deviation (SD)) or $\mathrm{n}(\%)$. Outcome measures were compared using chi-squared tests. A $p<0.05$ was considered to indicate statistical significance. Where missing data for some of the responses to the survey questions were encountered, those participants were excluded from some of the analysis, the number analysed (n) and percentage (\%) were quoted where pertinent. Statistical analysis was carried out using Qualtrics (Qualtrics, London, UK) and SPSS for Windows, Version 25.0 (IBM, Armonk, USA). ${ }^{7}$

A minimum sample size of 100 participants was required for $90 \%$ power, $\alpha=0.05$ and the ideal anticipated Cohen's ' $d$ ' effect size of 0.80 calculated using a web-based sample-size calculator (Raosoft, Seattle, USA). ${ }^{6}$ This sample size allows us to detect proportions within a margin error of $10 \%$ based on a $95 \%$ confidence interval.

\section{Results}

The study consisted of 110 respondents, of which, 32 were female and 78 were male. The demographics of the respondents are shown in Table 2.

Objective 1: To determine the compliance and awareness of PPE and the relevant equipment being used in different environments.

Out of 110 responding doctors, 100 (96\%) doctors were aware of the guidance and $92(88 \%)$ complied with the guidance on PPE set out by Public Health England (PHE).

Objective 2: To describe the common hygiene practices by UK doctors at the time of the COVID-19 pandemic

During a normal working shift in the hospital, half of the respondents (51\%) regularly washed their hands 10-20 times, with $35(35 \%)$ respondents washing their hands more than 20 times.

There is an equal distribution of responses in all three categories regarding the point at which clinicians wash their hands, with the significant response being 'after patient contact' (98\%).

A total of 91 (92\%) respondents stated that they changed their work clothes to scrubs before entering the clinical setting (Fig 1a). Similarly, 87 participants ( $88 \%$ ) reported changing back into their original attire from scrubs before returning home (Fig 1b).

A third of respondents $(n=34 ; 34 \%)$ replied that they would 'often' wear their surgical masks when not working in a specific ward, but 14 (14\%) of those had 'never' worn surgical masks when not working in a specific ward. Those working in COVID-19 areas had a higher proportion of individuals 'often' (AE_COVID $n=16$ $(33 \%)$; NAE_COVID $n=21(38 \%)$ ) or 'always' (AE_COVID $n=11$ (23\%); NAE_COVID n=10 (18\%)) wearing surgical masks. 
Table 2. Demographic data

\begin{tabular}{ll} 
Category & $\begin{array}{l}\text { Number of } \\
\text { participants }\end{array}$ \\
Gender, $\mathbf{n}$ & 78 \\
$\quad$ Male & 32 \\
Female & 110 \\
Total & \\
Working environment, $\mathbf{n}$ & 50 \\
AE_COVID & 60 \\
NAE_COVID & 19 \\
$\quad$ NON_COVID & 129 \\
Total & \\
Exposure to COVID-19, $\mathbf{n}$ (\%) & $100(94.34)$ \\
$\quad$ Direct exposure & $6(5.66)$ \\
Indirect exposure & $106(100)$ \\
Total & \\
Medical grade, $\mathbf{n}$ (\%) & $20(18.18)$ \\
Foundation year doctor (FY1-2) & $20(18.18)$ \\
Junior doctor (ST1-2 and IMT1-2) & $5(4.55)$ \\
Middle grade (staff grade/clinical assistants) & $25(22.73)$ \\
Specialist trainee (ST3-8) & $40(36.36)$ \\
Consultant & $110(100)$ \\
Total & \\
\hline AE_CovID = aerosolised environment; NAE_COVID = non-aerosolised \\
environment; NON_COVID = not in direct care of patients with COVID-19.
\end{tabular}

In NAE_COVID, only a small proportion of individuals also wore a fluid-resistant surgical apron along with a disposable plastic apron (fluid resistant surgical apron $n=22(38 \%)$ )

Regarding the use of footwear while seeing patients with COVID-19, 31 (36\%) responded that they wore 'normal work shoes',
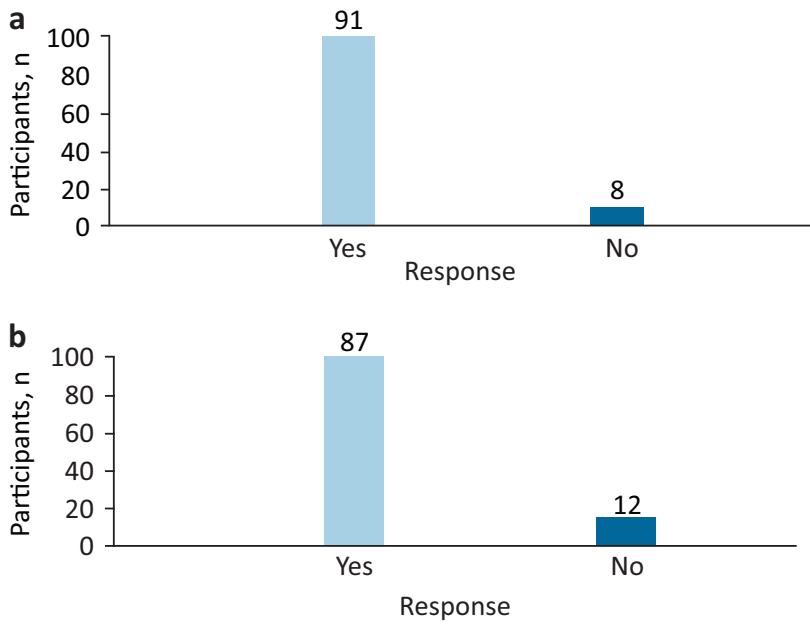

Fig 1. Overall responses for change of clothing while coming into the workplace and going home due to COVID-19 pandemic. a) Whether doctors changed from work clothing to scrubs before entering their respective clinical ward in the hospital. $n=99$. b) Whether doctors change back from scrubs to work clothing upon returning home. $\mathrm{n}=99$.
$13(15 \%)$ wore 'separate shoes' dedicated for hospital usage, 14 (16\%) wore 'surgical crocs', 22 (26\%) wore 'trainers', two (2\%) wore 'boots' and only four ( $5 \%$ ) used extra covering on their shoes. We measured the stress and anxiety levels of participants in bringing the infection home using a Likert scale marked from $0-5$ ( 0 being the lowest anxiety and 5 with the highest anxiety level), $52(53 \%)$ participants expressed a rating of $4-5$.

There was an equal distribution among participants in choosing to keep clothing in either a separate laundry basket, a dedicated bag or washed with hot water when asked about how they would wash their work clothing (mean $n=26(27 \%)$ ).

The majority (76 (78\%)) washed the scrubs that they wore for hospital 'every day' (Fig 3). Only seven (7\%) doctors washed their scrubs on a 'weekly basis'. Two-thirds (67 (69\%)) took off their work shoes before entering the house and 78 (80\%) doctors separated their work belongings in the household.

Upon returning home, the majority of doctors cleaned their 'stethoscope' and 'ID badge', with only those who worked directly with patients with COVID-19 (21) cleaning their 'credit/debit card' in their wallets as well. It is also important to consider that two individuals 'keep all items in a sealed waterproof bag' and five participants also clarified that the items 'are left at work'.

About the question on cleaning and washing, 82 (85\%) have said they took a shower 'upon returning home' instead of 'in the hospital' and five (5\%) had mentioned they would take a shower both in the hospital and upon returning home.

Only $11(11 \%)$ were able to keep a distance of two meters as a precaution when interacting with a family member at home.

\section{Objective 3: To compare the hygiene adaptations} shared by doctors by the working environment

Half of the respondents ( $52 \%$ ) cleaned their mobile phones regularly on leaving the wards with appropriate cleaning methods. Individuals in the NAE_COVID had the highest proportion of people cleaning their mobile phones $(n=32(59 \%))$.

Regarding the home adaptations, 35 (34\%) reported sleeping in a separate room away from their families. Doctors in the direct care of patients with COVID-19 reported a higher proportion of the use of a separate room to sleep in compared with those not routinely in direct care (total $n=36(87 \%)$ ).

Three-quarters of participants (76\%) reported using a separate bag for disposal of clothing for cleaning and 84 (88\%) participants in direct care for patients with COVID-19 reported a higher percentage of individuals disposing of their scrubs in a dedicated sealing bag after use.

Importantly, 46 (50\%) respondents washed their work attire at home at temperatures of $60-80^{\circ} \mathrm{C}$ (Fig 2). Only those in the direct care of patients with COVID-19 also used other temperature settings such as $0-59^{\circ} \mathrm{C}$ and $>80^{\circ} \mathrm{C}(n=44)$.

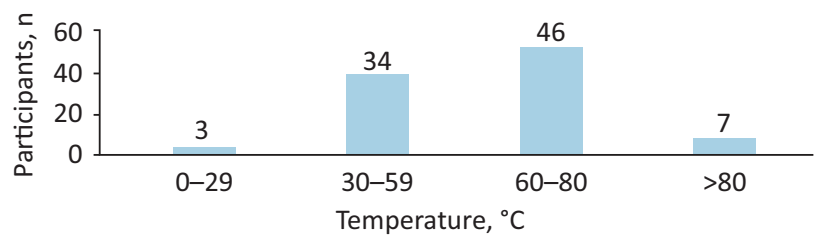

Fig 2. Washing machine temperature settings at home used by the doctors to wash their work clothing due to COVID-19 pandemic. $n=90$. 


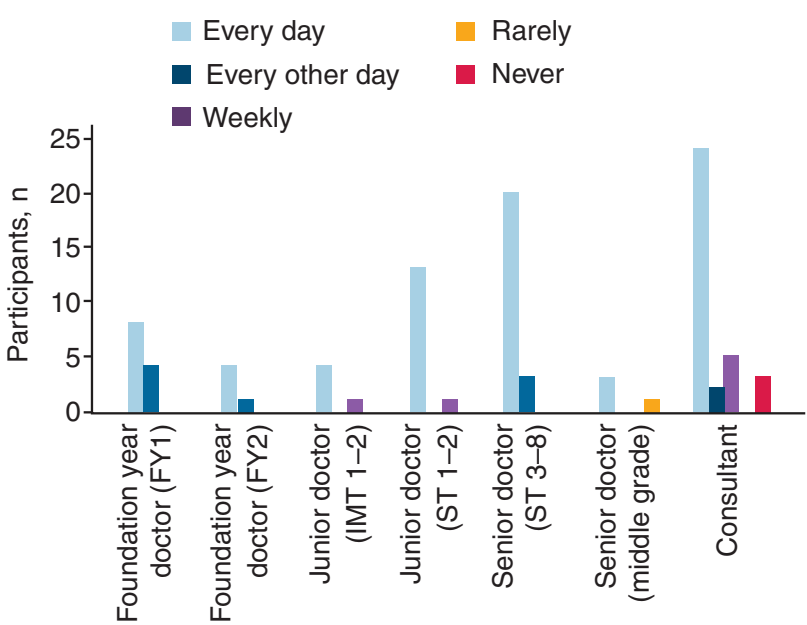

Fig 3. Responses of doctors regarding the frequency of washing their scrubs worn in the hospital. $n=97$.

Only 38 (39\%) participants wore surgical masks outside of the workplace. Those working in AE_COVID (40\%) or NAE_COVID (51\%) had a higher proportion of those willing to wear surgical masks outside the workplace compared to NON_COVID.

The adaptations to reduce the risk of infection to both doctors and their families were frequent hand washings (32\%) and having a shower before family interactions (16\%).

About $16 \%$ also mentioned frequent use of hand sanitisers and $16 \%$ took further precautionary measures to 'protect their families.' Interestingly three (5\%) participants also mentioned that they wiped their shoes by walking on the grass and two $(3 \%)$ participants encased their phone into 'cling film'.

Comparing practices of clinical grades, we found that a higher proportion of consultants and senior doctors washed their scrubs daily compared with junior doctors (Fig 3; junior doctors $n=29$ (within clinical grade $81 \%$ ), consultants and senior doctors $n=47$ (within clinical grade $77 \%$ )).

However, it was recognised that junior doctors were cleaning their stethoscopes more frequently when compared with the senior doctors and consultants (Fig 4a; junior doctors $n=25(68 \%)$; consultants and senior doctors $n=23(37 \%))$. The reason for this could be that junior doctors were examining these patients, more often than the consultants, therefore, they may clean their stethoscope more frequently due to the nature of their work.

A higher proportion of female doctors cleaned their belongings (pens; $72 \%$ vs $44 \%$ ) and their mobile phones ( $59 \%$ vs $31 \%$ ) regularly when compared with male doctors (Figs $4 \mathrm{~b}$ and $\mathrm{c}$ ). A possible reason could be that female doctors are generally more conscious about cleaning their items regularly and that they may be more worried about transmitting the COVID-19 infection onto others.

\section{Discussion}

After data analysis and survey completion, we could share the common hygiene practices of doctors both in secondary and tertiary care who are involved with patients with COVID-19.

We feel these practices are of great benefit to healthcare workers who are still dealing with COVID-19 cases at hospitals around the world or also in case of a second COVID-19 peak in various parts of the world, including the UK.
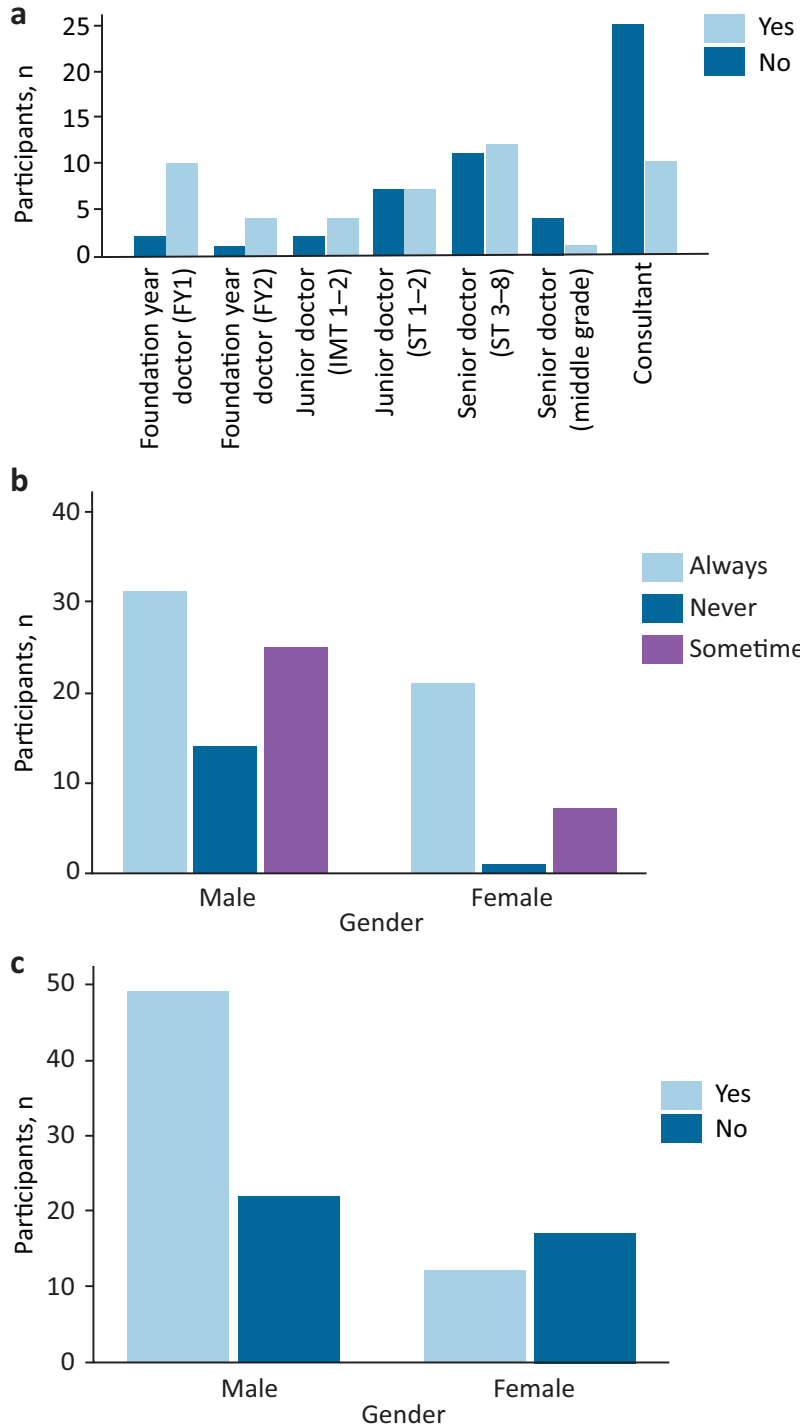

Fig 4. Response of doctors regarding cleaning commonly handled objects. a) Doctors who regularly cleaned their stethoscopes upon returning home. $n=100$. b) Doctors who tend to clean their mobile phones with any appropriate cleaning methods. $n=99$. c) Doctors who routinely clean work pens. $n=100$

Primarily, the majority of responses (86\%) from doctors were related to hand washing for at least 10-20 times and some more than 20 times during a normal 8-hour shift, we think this is a very important adaptation to highlight.

The Centers for Disease Control and Prevention (CDC) infection control guidelines stated that hand washing 'mechanically removes pathogens' and that the 'laboratory data demonstrates that the alcohol-based hand rub (ABHR) formulations' recommended have proven to 'inactivate SARS-CoV-2 ${ }^{8}{ }^{8}$ Therefore, we feel that this is one of the most important interventions in the fight against the spread of COVID-19.

The majority of responses in the study, (88-91\%) showed a very important adaptation, ie while coming into the hospital, the doctors would change from their normal attire into surgical scrubs before entering their respected wards. Similarly, we found doctors 
would change back into their normal clothing from scrubs before returning home from the hospital. We feel this is another useful adaptation as this virus commonly spreads through droplets and can also be aerosolised at times. This also prevents the chance of fomite transmission onto their clothes, therefore being carried home to spread the infection. By changing clothes daily this could lead to preventing the disease from spreading onto other people and their families. ${ }^{9}$

Most of the doctors understood the importance of the required PPE and complied with PHE recommendations in their work settings. The doctors also knew how to dispose of their work clothes into a red sealing bag after use. The majority of participants in the survey took a shower with hot water after reaching home, before having any family interaction. However, a few participants also took a shower at their workplace and some took one in both places. We feel that this may be another very important work adaptation to prevent the spread of COVID-19 because a hot shower could be potentially killing the virus on the human hairs and the skin. ${ }^{9}$

Another very important hygiene adaptation that we feel was significant to share was the temperature at which doctors washed their work clothing. In our survey, it was found that temperatures of $60-80^{\circ} \mathrm{C}$ and washing 'with hot water' were the optimum settings used by doctors to wash their clothes. Similarly, the majority of doctors also washed their scrubs, which were worn in the hospital daily. WHO state that temperatures at ' $56^{\circ} \mathrm{C}$ at around 10,000 units per 15 minutes' kills the virus. ${ }^{10}$ Therefore, we believe that adapting to using these optimum temperature settings regularly may reduce the duration of the virus staying on the surface of clinicians' clothes.

In this study, we also found items that were commonly cleaned by doctors were the stethoscope, ID badge, mobile phones and how these belongings were kept separately in the household. Heart \& Lung have published an article to remind healthcare workers to clean their stethoscopes regularly while sanitising their hands. ${ }^{11}$ They explain that stethoscopes have been shown to 'harbour potentially pathogenic bacteria' from the skin and to have 'nosocomial transmission of highly pathogenic viruses'.

This article strongly supports our findings that even small actions such as cleaning stethoscopes can vastly reduce the spread of COVID-19 between individuals.

The results of our study highlight that some doctors slept in a separate room away from the families at home and a few individuals were even staying in separate accommodation away from their families to limit the spread of COVID-19. In light of the social distancing measures, the CDC emphasises the importance of keeping a distance from others. They state that 'COVID-19 spreads mainly among people who are in close contact (within about 6 feet) for a prolonged period. ${ }^{12}$

Most participants in the survey expressed a high degree of concern and worry about potentially bringing the infection to their families. A review was conducted to analyse the current research that was focused on the impact COVID-19 has had on the mental health and stress levels of healthcare workers during the COVID-19 pandemic. There is increasing evidence that suggests COVID-19 is an 'independent risk factor for stress, anxiety and depression in some healthcare workers'.13 Therefore, the high anxiety levels that were shown by doctors in our study may further cause a detrimental impact on their mental health and well-being. This illustrates the importance of doctors to proactively adapt and learn from these common practices.

We noticed that junior doctors were cleaning their stethoscopes more frequently compared with senior doctors and consultants.
For clinical grade, we found that a higher proportion of junior doctors washed their scrubs daily compared with senior doctors and consultants. This is difficult to explain, but perhaps the junior doctors were more often in direct contact with patients compared with senior doctors or consultants.

It was also noted that female doctors were more meticulously concerned about cleaning their mobile phones and pens used in the wards compared with the male doctors. They may have been more anxious about bringing the infection home compared to their male counterparts.

However, there were some limitations in our study as we were not able to determine the COVID-19 infectivity status of the doctors completing the survey, thus it is difficult to establish if these were the best practices. Furthermore, we feel that there needs to be more work carried out where the healthcare workers' COVID-19 status or the antibody status can be tracked and looking similarly at their hygiene practices.

Our study is unique as it is the only study looking at particular adaptations in hygiene practises, both in the hospital and in the home environment across the whole of the UK. Thus, we feel strongly about sharing all the common practices, to where COVID-19 is rife and moving towards a peak, which may prove very useful for doctors and healthcare workers to know.

\section{Conclusion}

In some developing countries around the globe which are now facing a substantial rise in COVID-19 cases, it is crucial that healthcare workers can learn from these common hygiene adaptations of doctors in the UK during this pandemic. These common practices could potentially play an important role in reducing the spread of COVID-19 infection to the doctors as well as to their families, reducing morbidity and mortality of doctors and healthcare workers with this lethal virus. More studies may be needed on the subject of hygiene practices, but this research is still important to share with healthcare workers throughout the world.

\section{Summary}

\section{What is known?}

The coronavirus has a high transmissibility rate through means of respiratory droplets, direct contact and aerosolised particles. Doctors are at a higher risk of contracting and dying from the virus. Therefore, doctors are worried that they may spread the infection of COVID-19 onto themselves and to their family members at home from their working environments.

\section{What is the question?}

We carried out a qualitative survey to find out the common hygiene adaptations in hospitals and at home by doctors in the UK. These hygiene practices should be shared to other healthcare workers in an attempt to reduce the spread of infection onto themselves and their family, or in the unfortunate event of a second wave of the pandemic.

\section{What was found?}

The important hygiene practices we feel are useful for other healthcare workers to know about:

Hygiene adaptations in hospital

> The majority of doctors were aware of and complied to the standard PPE guidelines set by the PHE. 
Frequent hand washing of 10-20 times during a normal working shift.

> Changing from work clothing to scrubs before entering the clinical setting and similarly changing back before returning home.

> Some doctors wore separate shoes that are dedicated to the hospital.

> Around half of the participants cleaned their mobile phones after leaving the wards.

Hygiene adaptations at home:

$>$ Doctors expressed high levels of anxiety on the risk of spreading the infection onto their families.

> The majority of doctors routinely in the direct care of patients with COVID-19 slept in a separate room from their families at home.

> Doctors took off their work shoes before entering their home.

$>$ Doctors separated their work belongings in the household.

$>$ Doctors commonly chose to wash their clothing at a temperature of $60-80^{\circ} \mathrm{C}$ and with hot water.

> Doctors in the direct care of patients with COVID-19 disposed of their scrubs in a red sealing bag after use.

> Most doctors washed the scrubs that they wore for the hospital daily.

> Majority of doctors took a hot shower upon returning home before interacting with any family member.

What is the implication for the practice now?

These common practices are of great benefit for other healthcare workers to learn from. By adopting these practices, healthcare workers across the world could potentially be further reducing the risk of the spread of the COVID-19 infection, both to themselves and their families.

Also, by learning from these common practices, we can better prepare ourselves to potentially deal with an anticipated second wave of the coronavirus.

\section{Supplementary material}

Additional supplementary material may be found in the online version of this article at www.rcpjournals.org/clinmedicine:

S1 - Fuller dataset of questions and results of survey.

\section{Acknowledgements}

We would like to acknowledge the work of Dr Mustafa Hassan based in Coventry for his substantial support in conducing this survey.

\section{References}

1 Sariol A, Perlman S. Lessons for COVID-19 immunity from other coronavirus infections. Immunity 2020;53:248-63.

2 Garzaro G, Clari M, Ciocan C et al. COVID-19 infection and diffusion among the healthcare workforce in a large university-hospital in northwest Italy. Med Lav 2020;111:184-94.

3 World Health Organization. WHO Director-General's opening remarks at the media briefing on COVID-19 - 11 March 2020. WHO, 2020. www.who.int/dg/speeches/detail/who-directorgeneral-s-opening-remarks-at-the-media-briefing-on-covid-19—11march-2020 [Accessed 05 August 2020].

4 van Doremalen N, Morris DH, Holbrook MG et al. Aerosol and surface stability of SARS-CoV-2 as compared with SARS-CoV-1. N Engl J Med 2020:NEJMc2004973.

5 Qualtrics. Student survey. University of York, 2020. https://york. qualtrics.com/jfe/form/SV_3mm7s7WZWUvX6UB [Accessed 30 July 2020].

6 Raosoft. Sample size calculator. Raosoft, 2020. www.raosoft.com/ samplesize.html [Accessed 17 July 2020].

7 Qualtrics. Qualtrics for education. Qualtrics, 2020. www.qualtrics. com/uk/edu [Accessed 17 July 2020].

8 Centers for Disease Control and Prevention. Coronavirus disease 2019 (COVID-19): Hand hygiene recommendations. CDC, 2020. www.cdc.gov/coronavirus/2019-ncov/hcp/hand-hygiene.html [Accessed 14 July 2020].

9 Morawska L, Milton D. It is time to address airborne transmission of COVID-19. Clin Infect Dis 2020; ciaa939.

10 World Health Organization. Emergencies preparedness, response: First data on stability and resistance of SARS coronavirus compiled by members of WHO laboratory network. WHO, 2020. www.who. int/csr/sars/survival_2003_05_04/en [Accessed 17 July 2020].

11 Marinella MA. COVID-19 pandemic and the stethoscope: Do not forget to sanitize. Heart Lung 2020;49:350.

12 Centers for Disease Control and Prevention. Coronavirus disease 2019 (COVID-19): Social distancing. CDC, 2020. www.cdc.gov/ coronavirus/2019-ncov/prevent-getting-sick/social-distancing.html [Accessed 14 July 2020].

13 Spoorthy MS. Mental health problems faced by healthcare workers due to the COVID-19 pandemic-A review. Asian J Psychiatry 2020;51:102119.

Address for correspondence: Mr Syed Ammar Husain, Hull York Medical School, John Hughlings Jackson Building, University Road, Heslington, York YO10 5DD, UK. Email: hysh29@hyms.ac.uk 